\title{
Sentimento de angústia e isolamento social de universitários da área da saúde durante a pandemia da COVID-19
}

\section{Feeling of anguish and social isolation among undergraduate healthcare students during the COVID-19 pandemic}

\section{Sentimiento de angustia e aislamiento social de universitarios del área de la salud durante la pandemia de la COVID-19}

\author{
Aline Barbosa Teixeira Martins (iD \\ Universidade de Fortaleza - Fortaleza (CE) - Brasil \\ Cristina de Santiago Viana Falcão iD \\ Universidade de Fortaleza - Fortaleza (CE) - Brasil
}

Álvaro Magalhães Cavalcante Pereira (D)

Universidade de Fortaleza - Fortaleza (CE) - Brasil

Jannayna Queiroz Carvalho (iD

Universidade de Fortaleza - Fortaleza (CE) - Brasil

Jihane de Lima Diogo

Universidade de Fortaleza - Fortaleza (CE) - Brasil

Ygor Raphael Gomes Eloy (iD

Universidade de Fortaleza - Fortaleza (CE) - Brasil

Ana Paula Vasconcellos Abdon (iD

Universidade de Fortaleza - Fortaleza (CE) - Brasil

\section{RESUMO}

Objetivo: Identificar a prevalência do sentimento de angústia autorreferido e seus fatores relacionados, bem como a adesão ao isolamento social de universitários da área da saúde durante a pandemia da COVID-19. Métodos: Trata-se de um estudo transversal, desenvolvido em uma universidade privada e filantrópica, localizada no município de Fortaleza, Ceará, Brasil, com coleta de dados em junho 2020. Participaram 541 universitários ( $\geq 18$ anos) da área da saúde, que responderam um formulário eletrônico para coletar as variáveis demográficas relacionadas à pandemia da COVID-19, sentimentos de angústia e preocupação. Realizaram-se análises bivariada e multivariada para avaliar as relações com o desfecho sentimento de angústia. Resultados: Do total dos universitários, $89,5 \%(n=489)$ disseram estar angustiados, 90,4\% $(n=489)$ eram favoráveis ao isolamento social e 93,5\% $(n=503)$ aderiram ao isolamento social como combate à COVID19. Na análise multivariada, o sentimento de angústia relatado pelos universitários apresentou relação com a preocupação com o mundo $(O R=4,099 ; p=0,000)$. Conclusão: $O$ sentimento de angústia apresentado pelos universitários esteve relacionado ao sentimento de preocupação com o mundo e a serem predominantemente favoráveis ao isolamento social, e, por isso, aderiram a essa medida de enfrentamento do novo coronavírus.

Descritores: Estudantes de Ciências da Saúde; Infecções por Coronavirus; Emoções; Isolamento Social.

\section{ABSTRACT}

Objective: To identify the prevalence of self-reported anguish and related factors, as well as adherence to social isolation among undergraduate healthcare during the COVID-19 pandemic. Methods: This is a cross-sectional study conducted at a private and philanthropic university located in the municipality of Fortaleza, Ceará, Brazil, with data collected in June 2020. Participants were 541 undergraduate healthcare students ( $\geq 18$ years old) who completed an electronic form that collected demographic data relating to the COVID-19 pandemic and feelings of anguish and concern. Bivariate and multivariate analyses were performed to assess 
any correlations with the outcome 'feeling of anguish'. Results: Of all the university students, $89.5 \%$ ( $n=489)$ reported feeling anguished, $90.4 \%(n=489)$ were in favor of social isolation and 93.5\% ( $n=503)$ adhered to social isolation to fight COVID-19. In the multivariate analysis, the feeling of anguish reported by the university students was correlated with concern with the world $(O R=4.099 ; p=0.000)$. Conclusion: The feeling of anguish reported by the university students was correlated with concern with the world and with being predominantly in favor of social isolation, and hence they adhered to this measure for fighting the new coronavirus.

Descriptors: Health Science Students; Coronavirus Infections; Emotions; Social isolation.

\section{RESUMEN}

Objetivo: Identificar la prevalencia del sentimiento de angustia auto referido y sus factores relacionados así como la adhesión al aislamiento social de universitarios del área de la salud durante la pandemia de la COVID-19. Métodos: Se trata de un estudio transversal desarrollado en una universidad privada y filantrópica localizada en el municipio de Fortaleza, Ceará, Brasil con la recogida de datos en junio de 2020. Participaron 541 universitarios ( $\geq 18$ años) del área de la salud que han contestado un formulario electrónico para recoger datos de las variables demográficas relacionadas con la pandemia de la COVID-19, los sentimientos de angustia y la preocupación. Se realizó los análisis bivariado y multivariado para evaluar las relaciones con el desenlace sentimiento de angustia. Resultados: Del total de los universitarios, el 89,5\% ( $n=489)$ relataron estar angustiados, el 90,4\% (n=489) estaban de acuerdo al aislamiento social y el 93,5\% ( $n=503)$ adhirieron al aislamiento social para combatir la COVID19. En el análisis multivariado, el sentimiento de angustia relatado por los universitarios presentó relación con la preocupación con el mundo (OR=4,099; $p=0,000)$. Conclusión: El sentimiento de angustia presentado por los universitarios se relacionó con el sentimiento de preocupación con el mundo y ellos estaban favorables al aislamiento social y, por ello, adhirieron a esa medida de afrontamiento contra el nuevo coronavirus.

Descriptores: Estudiantes del Área de la Salud; Infecciones por Coronavirus; Emociones; Aislamiento Social.

\section{INTRODUÇÃO}

A Organização Mundial da Saúde (OMS) usou o termo "novo coronavírus 2019" para se referir a um coronavírus que afetava o trato respiratório inferior de pacientes com pneumonia em Wuhan, China, em 29 de dezembro de 2019, resultando na doença COVID-19(1-3).

A mesma organização declarou, em 30 de janeiro de 2020, que o surto da doença causada pelo novo coronavírus constitui uma emergência de saúde pública de importância internacional, o mais alto nível de alerta da Organização, conforme previsto no Regulamento Sanitário Internacional. Em 11 de março de 2020, a OMS declarou a pandemia do novo coronavírus ${ }^{(4)}$.

Com a duração e a gravidade da pandemia, a medida utilizada pela maioria das regiões do Brasil foi o distanciamento social ampliado (DAS), quando pessoas de todos os setores da sociedade precisam permanecer na residência enquanto durar a decretação da medida pelos gestores locais ${ }^{(5)}$. Diante dessa situação, além dos cuidados com as medidas de proteção para prevenir doenças respiratórias, as instituições de ensino públicas e particulares (escolas e universidades) aderiram ao DAS de acordo com as orientações do Ministério da Educação (ME) $)^{(6)}$.

No dia 13 de março de 2020, foi publicado um ato normativo respaldando, por meio da Portaria $n .^{\circ} 343$, de 17 de março de 2020, as instituições de educação superior (IES) para que, diante da pandemia da COVID-19, substituíssem as aulas presenciais por aulas em meios digitais enquanto durasse a situação(7).

Conforme solicitado pela Associação Brasileira de Mantenedoras de Ensino Superior (ABMES), está autorizado, em caráter excepcional, a utilização de meios e tecnologias para substituição das aulas presenciais pelo prazo inicial de 30 (trinta) dias durante a pandemia da COVID-19, podendo ser prorrogado conforme orientação do Ministério da Saúde (MS) ${ }^{(7)}$.

Os universitários, frente à modalidade de ensino remoto, necessitam sentir-se motivados e em um processo de autonomia pela busca do conhecimento ${ }^{(8)}$. Caso contrário, essa situação pode gerar sentimentos de angústia e solidão, levando-os a sentir-se desmotivados pela falta de interação, atenção e apoio por parte dos discentes e docentes $^{(9)}$. Essa realidade virtual reforça a necessidade das redes de apoio social durante esse período, visto que a falta de interações físicas e sociais são responsáveis por importantes impactos psicológicos negativos na saúde ${ }^{(10,11)}$.

A saúde mental é o equilíbrio emocional entre suas necessidades e as exigências ou vivências externas. É a capacidade de administrar a própria vida e as suas emoções em variadas situações, sem deixar de fazer o que é necessário. É entendida como estar de bem consigo e com os outros. Aceitar as exigências da vida, saber lidar com 
as emoções positivas e também com as negativas, como: alegria/tristeza; coragem/medo; amor/ódio; serenidade/ raiva; ciúmes; culpa; frustrações ${ }^{(12)}$.

Com a realização das aulas remotas (web aulas, videoconferências, entre outras), docentes da Universidade de Fortaleza (UNIFOR) aderiram ao sistema home office, dentro da sua carga horária de trabalho (horários de aula), utilizando aplicativos e o site da universidade para desenvolvimento das suas atividades, com agendamento prévio com os alunos (as). Perante a situação atual vivenciada em todos os setores e por todos os indivíduos, assim como também no meio acadêmico, o Programa Tutorial Acadêmico (PTA/CCS) da UNIFOR buscou promover estratégias para a promoção da saúde, com a intenção de cuidar da saúde dos alunos no âmbito físico, psíquico e social, pois entende-se que a saúde vai além da ausência de doença, mas engloba o mais completo bem-estar físico, mental e social. Para tanto, o setor adaptou seus projetos para o ambiente virtual, na tentativa de contemplar o completo bemestar físico, mental e social dos alunos, pois se sabe que as exigências e demandas da vida universitária evidenciam que o estudante universitário, desde o seu ingresso na instituição, deve apresentar recursos cognitivos e emocionais complexos para o manejo das demandas desse novo ambiente ${ }^{(13)}$. Para essa adaptação, fez-se necessário entender como os alunos estão vivenciando essa realidade atual, desenvolvendo suas atividades acadêmicas, atualizando-se em relação à situação atual e cuidando da sua saúde física e mental.

Esta pesquisa teve por objetivo identificar a prevalência do sentimento de angústia autorreferido e seus fatores relacionados, bem como a adesão ao isolamento social de universitários da área da saúde durante a pandemia da COVID-19.

\section{MÉTODOS}

Trata-se de um estudo transversal desenvolvido em uma universidade privada e filantrópica, localizada no município de Fortaleza, Ceará, Brasil, no período de junho de 2020.

Participaram 541 universitários (idade $\geq 18$ anos), matriculados em um dos cursos da área da saúde da instituição e com acesso à internet no período da coleta dos dados. Esse quantitativo foi estimado a partir de cálculo amostral, tendo como população os universitários da área da saúde $(n=3.500)$, prevalência de $50 \%$ (adotada por ser desconhecida), intervalo de confiança de $95 \%$ e erro amostral de $5 \%$.

O recrutamento dos participantes ocorreu por convite enviado pelo aplicativo de mensagens da instituição e em grupos de WhatsApp dos universitários pelo método bola de neve, que consiste em uma amostragem não probabilística em que os indivíduos selecionados convidam novos participantes da sua rede de amizade para responderem aos questionamentos. O processo se inicia com um pequeno número de indivíduos selecionados e vai crescendo à medida que novos participantes são convidados ${ }^{(14,15)}$. No convite havia uma explicação da pesquisa e um link de acesso ao Termo de Consentimento Livre e Esclarecido e ao formulário eletrônico da pesquisa no Google Forms $s^{\circledR}$.

O formulário foi elaborado a partir de perguntas fechadas, englobando as variáveis demográficas (faixa etária e residência); o curso de graduação; aspectos relacionados à pandemia da COVID-19 (conhecimento da doença, envolvimento com as notícias, isolamento social, rotina, impactos e estratégias para enfrentamento do isolamento social); sentimento de angústia e preocupação com o mundo e com o estado do Ceará.

Para avaliar a relação entre a variável dependente "sentimento de angústia" e as variáveis independentes, aplicouse o teste de qui-quadrado de Pearson, com os cálculos do odds ratio (OR) bruto e seus respectivos intervalos de confiança de 95\% (IC95\%), seguido pela regressão logística múltipla pelo método stepwise backward, utilizando o SPSS Statistics IBM ${ }^{\circledR}$ (versão 23.0). Para compor o modelo, realizou-se a seleção das variáveis com significância de $20 \%(p<0,20)$, ampliando as possibilidades de variáveis inseridas. Calcularam-se o OR ajustado e seus respectivos IC95\% no modelo final, adotando nível de significância de 5\%.

Este estudo foi aprovado pelo Comitê de Ética da UNIFOR, com Parecer n. ${ }^{\circ} 4.047 .430$, em obediência à Resolução n. ${ }^{\circ} 466 / 12$ e à Resolução $510 / 16$ do Conselho Nacional de Saúde ${ }^{(16)}$. Todos os participantes consentiram sua participação ao assinalar o item aceito como critério para continuidade na pesquisa.

\section{RESULTADOS}

Do total de 541 universitários, houve maior proporção da faixa etária 19 a 29 anos $(66,9 \% ; n=362)$ e residência fixada em Fortaleza (85,4\%; n=462). Sobre o curso de graduação, $170(31,4 \%)$ eram alunos do curso de Educação Física, seguidos por $87(16,1 \%)$ do curso de Psicologia e 60 (11,1\%) do curso de Estética e Cosmética (Tabela I). 
Tabela I - Distribuição das variáveis demográficas, curso de graduação e aspectos relacionados a pandemia da COVID-19 dos universitários da área da saúde. Fortaleza, Ceará, 2020.

\begin{tabular}{|c|c|c|}
\hline Variáveis & Frequência & Percentual (\%) \\
\hline \multicolumn{3}{|l|}{ Demográficos } \\
\hline \multicolumn{3}{|l|}{ Faixa etária (anos) } \\
\hline Até 18 & 84 & 15,5 \\
\hline Entre 19 a 29 & 362 & 66,9 \\
\hline Acima de 29 & 95 & 17,6 \\
\hline \multicolumn{3}{|l|}{ Residência } \\
\hline Fortaleza & 462 & 85,4 \\
\hline Região metropolitana e interior & 79 & 14,6 \\
\hline \multicolumn{3}{|l|}{ Curso de graduação } \\
\hline Educação Física & 170 & 31,4 \\
\hline Enfermagem & 28 & 5,2 \\
\hline Estética e Cosmética & 60 & 11,1 \\
\hline Farmácia & 17 & 3,1 \\
\hline Fisioterapia & 22 & 4,1 \\
\hline Fonoaudiologia & 11 & 2,0 \\
\hline Medicina & 32 & 5,9 \\
\hline Medicina Veterinária & 20 & 3,7 \\
\hline Odontologia & 54 & 10,0 \\
\hline Psicologia & 38 & 7,0 \\
\hline Não informado & 89 & 16,5 \\
\hline \multicolumn{3}{|c|}{$\begin{array}{l}\text { Aspectos relacionados a pandemia da COVID-19 } \\
\text { Conhecimento da doença }\end{array}$} \\
\hline Suficiente & 320 & 59,1 \\
\hline Insuficiente & 220 & 40,7 \\
\hline \multicolumn{3}{|l|}{ Envolvimento com as notícias } \\
\hline Sim & 508 & 93,9 \\
\hline Não & 33 & 6,1 \\
\hline \multicolumn{3}{|l|}{ Favorável ao isolamento social } \\
\hline Sim & 489 & 90,4 \\
\hline Não & 52 & 9,6 \\
\hline \multicolumn{3}{|l|}{ Adesão ao isolamento social } \\
\hline Sim & 503 & 93,5 \\
\hline Não & 35 & 6,5 \\
\hline \multicolumn{3}{|l|}{ Impactos causados na sua vida\# } \\
\hline Emocional & 369 & 68,3 \\
\hline Financeiro & 374 & 69,1 \\
\hline Saúde & 206 & 38,0 \\
\hline Formação Educacional & 410 & 75,7 \\
\hline Relações Interpessoais & 153 & 28,2 \\
\hline \multicolumn{3}{|c|}{ Impactos causados na vida de outras pessoas" } \\
\hline Emocional & 479 & 88,5 \\
\hline Financeiro & 527 & 97,4 \\
\hline Saúde & 431 & 79,6 \\
\hline Formação Educacional & 362 & 66,9 \\
\hline Relações Interpessoais & 263 & 48,6 \\
\hline \multicolumn{3}{|l|}{ Estratégias adotadas" } \\
\hline Rotina de estudos virtuais & 387 & 71,5 \\
\hline Atividade física em casa & 286 & 52,8 \\
\hline Atividades domésticas & 177 & 32,7 \\
\hline Atividades extras de relaxamento & 177 & 32,7 \\
\hline Cuidados com filhos/familiares & 66 & 12,1 \\
\hline Outros* & 29 & 5,3 \\
\hline Não utilizaram estratégias & 5 & 0,9 \\
\hline \multicolumn{3}{|l|}{ Sentimento de angústia } \\
\hline Sim & 484 & 89,5 \\
\hline Não & 57 & 10,5 \\
\hline \multicolumn{3}{|l|}{ Preocupação com o mundo } \\
\hline Sim & 496 & 91,7 \\
\hline Não & 45 & 8,3 \\
\hline \multicolumn{3}{|l|}{ Preocupação com o Ceará } \\
\hline Sim & 494 & 91,3 \\
\hline Não & 47 & 8,7 \\
\hline
\end{tabular}

\#variável categórica não excludente 
Sobre os aspectos relacionados à pandemia da COVID-19, 320 (59,3\%) disseram ter conhecimento suficiente sobre a doença, $508(93,9 \%)$ tinham envolvimento com as notícias, $489(90,4 \%)$ eram favoráveis ao isolamento social e $503(93,5 \%)$ aderiram ao isolamento social como combate à COVID-19. Os impactos na formação educacional $(75,7 ; n=410)$ e financeiros $(69,1 \% ; n=374)$ foram o mais referidos na vida dos universitários. Sobre os impactos na vida de outras pessoas, os financeiros $(97,4 \% ; n=527)$ e o emocional $(88,5 \% ; n=479)$ foram os mais citados. A rotina de estudos virtuais $(71,5 \% ; n=387)$ e as atividade físicas em casa $(52,8 \% ; n=286)$ eram as estratégias mais adotadas no enfrentamento ao isolamento social.

Acerca dos sentimentos, $489(89,5 \%)$ disseram estar angustiados, $496(91,7 \%)$ estavam preocupados com a pandemia em relação ao mundo e 494 (91,3\%) em relação ao Ceará (Tabela II).

Constatou-se, na análise bivariada, que o sentimento de angústia estava associado ao envolvimento com as notícias ( $O R=2,466 ; p=0,039)$, à preocupação com o mundo $(O R=4,173 ; p=0,000)$ e à preocupação com o Ceará $(O R=3,910 ; p=0,000)$ (Tabela II). Contudo, na análise multivariada, o sentimento de angústia relatado pelos universitários apresentou relação somente com a preocupação com o mundo $(O R=4,099 ; p=0,000)$, sendo o sentimento de angústia quatro vezes maior naqueles preocupados com o mundo (Tabela III).

Tabela II - Análise bivariada do sentimento de angústia dos universitários durante a pandemia da COVID-19. Fortaleza, Ceará, 2020.

\begin{tabular}{|c|c|c|c|c|}
\hline \multirow{2}{*}{ Variável } & \multicolumn{2}{|c|}{ Sentimento de angústia } & \multirow{2}{*}{ OR bruto (IC95\%) } & \multirow{2}{*}{ p-valor } \\
\hline & Não n (\%) & $\operatorname{Sim} n(\%)$ & & \\
\hline Faixa etária (anos) & & & & 0,748 \\
\hline Até 18 & $9(15,8)$ & $75(15,5)$ & 1 & \\
\hline Entre 19 a 29 & $36(63,2)$ & $326(67,4)$ & $1,087(0,502-2,352)$ & \\
\hline Acima de 29 & $12(21,1)$ & $83(17,1)$ & $0,830(0,331-2,081)$ & \\
\hline Residência & & & & $0,086^{a}$ \\
\hline Região metropolitana e interior & $4(7,0)$ & $75(15,5)$ & 1 & \\
\hline Fortaleza & $53(93,0)$ & $409(84,5)$ & $0,412(0,145-1,171)$ & \\
\hline Conhecimento da doença & & & & 0,825 \\
\hline Suficiente & $33(57,9)$ & $287(59,4)$ & 1 & \\
\hline Insuficiente & $24(42,1)$ & $196(40,6)$ & $0,939(0,538-1,638)$ & \\
\hline Envolvimento com notícias & & & & $0,039^{a}$ \\
\hline Não & $7(12,3)$ & $26(5,4)$ & 1 & \\
\hline Sim & $50(87,7)$ & $458(94,6)$ & $2,466(1,019-5,970)$ & \\
\hline Favorável ao isolamento social & & & 0,497 & 0,470 \\
\hline Não & $7(12,3)$ & $45(9,3)$ & & \\
\hline $\operatorname{Sim}$ & $50(87,7)$ & $439(90,7)$ & $1,366(0,585-3,190)$ & \\
\hline Adesão ao isolamento social & & & & 0,337 \\
\hline Não & $2(3,5)$ & $33(6,8)$ & 1 & \\
\hline Sim & $55(96,5)$ & $451(93,2)$ & $0,497(0,116-2,128)$ & \\
\hline Preocupação com o Mundo & & & & $0,000^{*}$ \\
\hline Não & $13(22,8)$ & $32(6,6)$ & 1 & \\
\hline Sim & $44(77,2)$ & $452(93,2)$ & $4,173(2,041-8,532)$ & \\
\hline Preocupação com o Ceará & & & & $0,000^{*}$ \\
\hline Não & $13(22,8)$ & $34(7,0)$ & 1 & \\
\hline Sim & $44(77,2)$ & $450(93,0)$ & $3,910(3,910-7,955)$ & \\
\hline
\end{tabular}

OR: odds ratio; IC95\%: intervalo de confianças de $95 \%$. ${ }^{*} \mathrm{p}<0,05$; ${ }^{a}$ variável selecionada para o modelo de regressão logística

Tabela III - Análise multivariada do sentimento de angústia dos universitários durante a pandemia da COVID-19. Fortaleza, Ceará, 2020.

\begin{tabular}{lcc}
\hline \multirow{2}{*}{ Variável } & Sentimento de angústia & p-valor \\
\cline { 2 - 3 } & OR ajustado (IC95\%) & 0,113 \\
\hline Residência & $0,422(0,145-1,225)$ & 0,332 \\
Envolvimento com as notícias & $1,625(0,610-4,329)$ & $0,000^{*}$ \\
Preocupação com o mundo & $4,099(1,998-8,408)$ & 0,227 \\
Preocupação com o Ceará & $1,806(0,673-5,281)$ & \\
\hline
\end{tabular}

OR=odds ratio; Modelo de Regressão Logística: $X 2=16,167$; Cox\&Snell=0,029; Nagelkerke: 0,060; * $<<0,05$ 


\section{DISCUSSÃO}

O presente estudo evidenciou que universitários dos cursos da saúde de uma IES se encontravam angustiados e preocupados com a situação estadual, e, principalmente, mundial, relacionada aos impactos da Covid-19 nos aspectos financeiros, emocionais e na formação educacional.

Para atender a essas demandas, a Política Nacional de Promoção à Saúde (PNPS) se baseia no conceito ampliado de saúde e apresenta sua promoção como um conjunto de estratégias e formas de produzir saúde, no âmbito individual e coletivo, com responsabilidades para os três entes federados ${ }^{(17)}$

Durante a pandemia, a PNPS reforça a importância de uma política transversal, integral e intersetorial, promovendo a qualidade de vida e reduzindo a vulnerabilidade e riscos à saúde relacionados aos fatores determinantes e condicionantes. Parâmetros Curriculares Nacionais orientam sobre a necessidade de que as concepções sobre saúde, a valorização de hábitos e estilos de vida, e as atitudes perante as diferentes questões relativas à saúde perpassem todas as áreas de estudo, de modo que possam processar-se regularmente e de modo contextualizado no cotidiano da experiência escolar e/ou, no caso do presente estudo, na vida universitária ${ }^{(18)}$.

Em setembro de 2017, mais de 600 pessoas morreram em decorrência do novo coronavírus na Arábia Saudita. Diante dessa realidade, realizouse uma pesquisa com o objetivo de investigar se o Ministério da Saúde da Arábia Saudita havia implementado as melhores práticas de relações públicas durante uma grande crise de saúde ou não. Para obtenção desses dados, aplicou-se uma pesquisa em universidades, com 875 estudantes, com idade variando entre 18 e 45 anos, com $30 \%$ dos participantes se formando em um setor relacionado à saúde ${ }^{(19)}$.

Sabe-se que, no período de pandemia, as informações recebidas e, em especial, a quarentena vivenciada, tendem a aumentar o sofrimento mental na população em geral em decorrência do isolamento social e da limitação da comunicação interpessoal, destacando a impossibilidade de convívio com parentes e entes queridos ${ }^{(20)}$. Esses dados são reforçados no presente estudo quando os participantes referem estar angustiados com a situação no período de pandemia no Ceará e no mundo.

Durante a crise atual, a angústia mental resultou em várias consequências comportamentais, como estresse, ansiedade, medo, raiva e depressão, culminando em situações extremas e comportamentos suicidas ${ }^{(21-24)}$.

Estudo realizado na China reforça a situação apresentada quando, em última amostra nacional, indicou que a prevalência de qualquer distúrbio (excluindo demência) resultava em transtornos de ansiedade e transtornos depressivos ${ }^{(25)}$. Em Wuhan, houve prevalência muito mais alta de depressão, ansiedade e da combinação de ambas ${ }^{(26)}$.

Com o final de uma pandemia, o impacto se apresenta durante e após o término dela, com consequências econômicas, sociais e culturais geradas ou agravadas. Uma pesquisa que avaliou as consequências psicológicas da quarentena em epidemias recentes identificou a presença de efeitos negativos, como irritabilidade, humor deprimido, confusão, raiva, insônia, exaustão emocional e sintomas de estresse pós-traumático ${ }^{(27)}$. Essa preocupação foi apresentada de forma local e nacional pelos participantes.

Seguindo as questões emocionais relatadas pela grande maioria dos alunos, um estudo realizado em 2009 sobre a pandemia de H1N1 comparou estudantes de graduação que estavam em quarentena com aqueles que não estavam em quarentena imediatamente após o período de quarentena, e não encontraram diferença significativa entre os grupos em termos de sintomas de estresse póstraumático ou problemas gerais de saúde mental(28)

Ressalta-se que o estudo acima citado ${ }^{(28)}$ foi realizado em período de uma pandemia mais leve do que a pandemia do momento atual, o que possivelmente não resultou em grandes impactos na saúde mental. A quarentena mais longa está associada a piores resultados, considerando que os participantes ficarão mais expostos aos agentes estressores ${ }^{(29)}$.

Além dos aspectos emocionais, a China, primeiro país a enfrentar a pandemia, registrou o maior declínio na sua produção industrial desde os anos 90 , refletindo nos aspectos financeiros, resultando na diminuição drástica das exportações, atingindo a cadeia produtiva de indústrias de muitos países ${ }^{(30,31)}$. Esses dados corroboram os achados de pesquisa que aponta o impacto na situação financeira pessoal $(69,1 \%)$ e da população em geral $(97,4 \%)$.

Diante dos resultados apresentados, a Universidade de Fortaleza desenvolveu estratégias, por meio de projetos virtualizados, na tentativa de amenizar o sofrimento apresentado pelos participantes durante o período de isolamento social, tais como: "Conexão CCS", com palestras mensais, "Roda de conversa: Como lidar com ansiedade em tempos difíceis" (realizada quinzenalmente) e "Roda de conversa: Dialogando sobre o preconceito na Universidade" (realizada quinzenalmente), palestras sobre liderança acadêmica, o projeto \#desacelera, com vídeos de meditação, orientações posturais e alimentação, além de atendimento virtual, entre outras.

O "Conexão CCS" foi realizado por meio de lives no Instagram e no Google Meet com temáticas abordando gestão do tempo em tempo de pandemia, estratégias para aprendizagem em ambientes virtuais, entre outros assuntos. $\mathrm{Na}$ 
roda de conversa sobre ansiedade, a mediação foi realizada por uma psicóloga, e os alunos eram sensibilizados com músicas e imagens, depois era dado espaço para a fala deles, assim como na roda "Dialogando sobre o preconceito na universidade". O projeto \#desacelera, como apresentado anteriormente, disponibilizou vídeos no Instagram.

O estudo vem contribuir com a população e comunidade científica, evidenciando que a pandemia pelo novo coronavírus afetou os estudantes com um sentimento de incerteza e angústia em decorrência da preocupação com a doença em nível local e nacional. Recomenda-se que, ao ser detectado sentimentos dessa grandeza, os indivíduos possam ter um acompanhamento profissional, a fim de ajudar a transcorrer essa fase de forma mais tranquila, motivo pelo qual a instituição esteve próxima dos alunos e desenvolvendo projetos que os auxiliassem nessa fase difícil.

A atual pesquisa apresentou algumas limitações, principalmente em relação à participação representativa de alunos de todos os cursos do CCS, pois, como o recrutamento foi realizado por meio de torpedos institucionais e do Whatsapp, resultou em uma amostra aleatória, sem representação de acordo com o quantitativo de alunos/curso.

\section{CONCLUSÃO}

Os universitários analisados apresentaram sentimento de angústia relacionado ao sentimento de preocupação com o mundo e a serem predominantemente favoráveis ao isolamento social, e, por isso, aderiram a essa medida de enfrentamento do novo coronavírus.

\section{CONTRIBUIÇÕES}

Aline Barbosa Teixeira Martins, Cristina de Santiago Viana Falcão e Ana Paula Vasconcellos Abdon contribuíram com a elaboração e delineamento do estudo; aquisição, análise e interpretação de dados; redação e/ou revisão do manuscrito. Álvaro Magalhães Cavalcante Pereira contribuiu com a redação e/ou revisão do manuscrito. Jannayna Queiroz Carvalho, Jihane de Lima Diogo e Ygor Raphael Gomes Eloy contribuíram com a elaboração e delineamento do estudo.

\section{CONFLITOS DE INTERESSE}

Os autores afirmam que não houve conflitos de interesse na execução desta pesquisa.

\section{REFERÊNCIAS}

1. Li Q, Guan X, Wu P, Wang X, Zhou L, Tong Y, et al. Early transmission dynamics in Wuhan, China, of novel coronavirus-infected pneumonia. N Engl J Med [Internet]. 2020 [acesso em 2020 Abr 8]. doi: 10.1056/ NEJMoa2001316

2. Centers for Disease Control and Preventio. 2019 Novel coronavirus, Wuhan, China [Internet]. 2020 [acesso em 2020 Fev 1]. Disponível em: https://www.cdc.gov/ coronavirus/2019-nCoV/summary.html

4. Organização Pan-Americana da Saúde Brasil. Folha informativa - COVID-19 (doença causada pelo novo coronavírus) [Internet]. 2020 [acesso em 2020 Abr 8]. Disponível em: https://www.paho.org/bra/index. php?option=com_content\&view $=$ article $\&$ id $=6101$ : covid $19 \&$ ltemid $=875$

5. Ministério da Saúde. Ministério da Saúde define critérios de distanciamento social [Internet]. 2020 [acesso em 2020 Abr 8]. Disponível em: https://www.saude.gov.br/noticias/agencia-saude/46666-ministerio-da-saudedefine-criterios-de-distanciamento-social

6. Ministério da Saúde. Todos pela educação: nota técnica Ensino a distância na educação básica frente à pandemia da COVID-19 [Internet]. 2020 [acesso em 2020 Abr 8]. Disponível em: https://crianca.mppr.mp.br/ arquivos/File/publi/todos_pela_educacao/nota_tecnica_ensino_a_distancia_todospelaeducacao_covid19.pdf

7. Associação Brasileira de Mantenedoras de Ensino Superior. MEC atende solicitação da ABMES e define regras para aulas em meios digitais durante o coronavírus [Internet]. 2020 [acesso em 2020 Abr 8]. Disponível em: https://abmes.org.br/noticias/detalhe/3670/mec-atende-solicitacao-da-abmes-e-define-regraspara-aulas-em-meios-digitais-durante-o-coronavirus

8. Ishida JS, Stefano SR, Andrade SM. Avaliação da satisfação no ensino de pós à distância: a visão dos tutores e alunos do PNAP/UAB. Avaliação (Campinas) [Internet]. 2013 [acesso em 2020 Set 22];18(3):749- 


\section{2. doi: $10.1590 / \mathrm{S} 1414-40772013000300012$}

9. Dosea GS, Rosario RWS, Silva EA, Firmino LR. Métodos ativos de aprendizagem no ensino online: a opinião de universitários durante a pandemia de covid-19. Educação [Internet]. 2020 [acesso em 2020 Set 22];10(1). Disponível em: https://periodicos.set.edu.br/educacao/article/view/9074/4134

10. Centers for Disease Control and Prevention. Social distancing, quarantine, and isolation: keep your distance to slow the spread [Internet]. 2020 [acesso em 2020 Set 22]. Disponível em: http://www.cdc.gov/ coronavirus/2019-ncov/prevent-getting-sick/social-distancing.html

11. European Centre for Disease Prevention and Control. Considerations relating to social distancing measures in response to COVID-19: second update [Internet]. 2020 [acesso em 2020 Abr 8]. Disponível em: http://www. ecdc.europa.eu/sites/default/files/documents/covid-19-social-distancing-measuresg-guide-second-update.pdf

12. Secretaria do Estado da Saúde do Paraná. Definição de Saúde Mental [Internet]. 2020 [acesso em 2020 Abr 8]. Disponível em: http://www.saude.pr.gov.br/modules/conteudo/conteudo.php?conteudo=1059

13. Padovani RC, Neufeld AB, Maltoni J, Barbosa LNF, Souza WF, Cavalcanti HAF, et al. Vulnerabilidade e bem-estar psicológicos do estudante universitário. Rev Bras Ter Cogn [Internet]. 2014 [acesso em 2020 Jul 14];10(1):2-10. Disponível em: http://www.rbtc.org.br/detalhe_artigo.asp?id=188\#

14. Coleman JS. Snowball sampling: problems and thecniques of chain referral sampling. Hum Organ. 1958;17:28-36.

15. Goodman LA. Snowball sampling. Ann Math Sta. 1961;32:148-70.

16. Brasil. Conselho Nacional de Saúde. Resolução CNS n. 466, de 12 de Dezembro de 2012. Aprova diretrizes e normas regulamentadoras de pesquisa envolvendo seres humanos. Diário Oficial da União: seção 1, Brasília, DF, n 12, p 59, 13 Jun 2012.

17. Dias MAS, Oliveira IP, Silva LMS, Vasconcelos MIO, Machado MFAS, Forte FDS, et al. Política Nacional de Promoção da Saúde: um estudo de avaliabilidade em uma região de saúde do Brasil. Ciênc Saúde Colet [Internet]. 2018 [acesso em 2020 Jul 13];23(1):103-14. Disponível em: https://scielosp.org/pdf/csc/2018. v23n1/103-114/pt

18. Ministério da Saúde (BR). Politica Nacional de Promoção à Saúde [Internet]. $3^{\mathrm{a}}$ ed. Brasília: Ministério da Saúde; 2010 [acesso em 2020 Jul 14]. Disponível em: https://bvsms.saude.gov.br/bvs/publicacoes/politica_ nacional_promocao_saude_3ed.pdf

19. Alsulaiman AS. Health crisis in the Kingdom of Saudi Arabia: a study of saudis' knowledge of Coronavirus, attitudes toward the ministry of health's coronavirus preventive campaigns, and trust in coronavirus messages in the media [dissertação] [Internet]. 2018 [acesso em 2020 Maio 4]. Disponível em: http://rave.ohiolink.edu/ etdc/view?acc_num=bgsu1521673786522563

20. Oliveira DS, Firmo AC, Bezerra IC, Leite JHC. COVID-19: do enfrentamento ao fortalecimento de estratégias em saúde mental - uma revisão narrativa. Reflexões Bioét [Internet]. 2020 [acesso em 2020 Jun 9];1(4). Disponível em: fhttps://escsresidencias.emnuvens.com.br/hrj/article/view/34/28

21. Ahmed MZ, Ahmed O, Aibao Z, Hanbin S, Siyu L, Ahmad A. Epidemic of COVID-19 in China and associated psychological problems. Asiaa J Psychiatr [Internet]. 2020 [acesso em 2020 Jun 30];102092. doi: 10.1016/j. ajp.2020.102091

22. Bhuiyan AI, Sakib N, Pakpour A, Griffiths MD, Mamun MA. COVID-19-related suicides in Bangladesh due to lockdown and economic factors: case study evidence from media reports. Int J Ment Health Addict [Internet]. 2020 [acesso em 2020 Jun 30]:1-16. Disponível em: https://www.ncbi.nlm.nih.gov/pmc/articles/PMC7228428/

23. Griffiths MD, Mamun MA. COVID-19 suicidal behavior among couples and suicide pacts: case study evidence from press reports. Psychiatry Res [Internet]. 2020 [acesso em 2020 Jun 30];289:113105. doi: 10.1016/j. psychres. 2020

24. Mamun MA, Griffiths MD. First CONVID -19 suicide case in Bangladesh due fear of COVID-19 and xenofobia: possible suicide prevention strategies. Asian J Psychiatr [Internet]. 2020 [acesso em 2020 Jun 30];51:102073. doi: 10.1016/j.ajp.2020.102073 
25. Huang Y, Wang Y, Wang HH, Zhaorui Liu Z, Yu X, Jie Yan J, et al. Prevalence of mental disorders in China: a cross-sectional epidemiological study. Lancet Psychiatry [Internet]. 2019 [acesso em 2020 Jun 30];6(3):21124. Disponível em: https://pubmed.ncbi.nlm.nih.gov/30792114/

26. Gao J, Zheng P, Jia Y, Chen H, Mao Y, Chen S, et al. Mental health problems and social media exposure during COVID-19 outbreak. Plos One [Internet]. 2020 [acesso em 2020 Jun 30];16:1-10. Disponível em: https://journals. plos.org/plosone/article/file?id=10.1371/journal. pone.0231924\&type=printable

27. Fundação Oswaldo Cruz. Saúde mental e atenção psicossocial na pandemia COVID-19: recomendações para gestores [Internet]. [2020] [acesso em 2020 Abr 23]. Disponível em: https://portal.fiocruz.br/documento/ saude-mental-e-atencao-psicossocial-na-pandemia-covid-19-recomendacoes-para-gestores

28. Wang $Y, X u B, Z$ hao G, Cao R, He X, Fu S, et al. Is quarantine related to immediate negative psychological consequences during the 2009 H1N1 epidemic? Gen Hosp Psychiatry [Internet]. 2011 [acesso em 2020 Jun 10];33:75-7. Disponível em: https://pubmed.ncbi.nlm.nih.gov/21353131/

29. Brooks SK, Webster RK, Smith LE, Woodland L, Wessely S, Greenberg N, et al. The psychological impact of quarantine and how to reduce it: rapid review of the evidence. Lancet [Internet]. 2020 [acesso em 2020 Jul 13];395(10227):912-20. Disponível em: https://www.thelancet.com/journals/lancet/article/PIIS01406736(20)30460-8/fulltext

30. Wang C, Pan R, Wan X, Tan Y, Xu L, Ho C, et al. Immediate Psychological Responses and Associated Factors during the Initial Stage of the 2019 Coronavirus Disease (COVID-19) Epidemic among the General Population in China. Int J Environ Res Public Health [Internet]. 2020 [acesso em 2020 Jun 30];17(5):1-25. Disponível em: https://pubmed.ncbi.nlm.nih.gov/32155789/

31. Lin ECL, Peng YC, Tsai JCH. Lessons learned from the anti-SARS quarantine experience in a hospital-based fever screening station in Taiwan. Am J Infect Control [Internet]. 2010 [acesso em 2020 Jun 30];38(4):302-7. Disponível em: https://www.ncbi.nlm.nih.gov/pmc/articles/PMC7115330/

\section{Endereço para correspondência:}

Aline Barbosa Teixeira Martins

Universidade de Fortaleza - Curso de Estética e Cosmética

Av. Washington Soares, 1321

Bairro: Edson Queiroz

CEP: 60811-905 - Fortaleza - CE - Brasil

E-mail: alinebate@unifor.br

Como citar: Martins ABT, Falcão CSV, Pereira AMC, Carvalho JQ, Diogo JL, Eloy YRG, et al. Sentimento de angústia e isolamento social de universitários da área da saúde durante a pandemia da COVID-19. Rev Bras Promoç Saúde. 2020;33:11444. 